\title{
Conjugacy, Involutions, and Reversibility for Real Homeomorphisms
}

\author{
ANTHONY G. O'FARRELL ${ }^{1}$
}

\begin{abstract}
The classification up to conjugacy of the homeomorphisms of the real line onto itself is well-understood by the experts, but there does not appear to be an exposition in print. In other words, it is mathematical folklore. In this expository paper, we give a complete but concise account of the classification, in terms of a suitable topological signature concept. A topological signature is a kind of pattern of signs. We provide similar classifications for homeomorphisms that fix a given subset, and for germs of homeomorphisms at a point. For direction-reversing homeomorphisms, we show that the signature of the compositional square is antisymmetric. We go on to apply the conjugacy classification and signatures to reprove recent results of Jarczyk on the composition of involutions. His results classify the reversible homeomorphisms (the composition of two involutions), and show that each homeomorphism is the composition of at most four involutions. The reversible direction-preserving homeomorphisms have symmetric signatures.
\end{abstract}

\section{INTRODUCTION}

Interest in reversible maps was motivated by the observation that dihedral groups of maps arise in diverse applications (such as the $n$-body problem, billiards, real and complex analysis, approximation theory, conformal mapping, linear algebra and analytic geometry, cf. [1]). People such as Poincaré and Birkhoff exploited reversibility in the context of classical Hamiltonian systems. The reversible nature of such systems helps in the investigation of their periodic orbits. Smale's student Devaney [2] initiated the formal study of

\footnotetext{
${ }^{1}$ Supported by EU Research Training Network Contract no. HPRN-CT-200000116.

2000 Mathematics Subject Classification. Primary 57S25, Secondary 22E65.
} 
smooth reversible systems, not necessarily derived from a Hamiltonian, and there has been considerable work on such systems. The main focus has been on higher dimensions, and it is only in this century that attention turned to the simplest case: maps in one real variable. In recent papers, Jarczyk [4, 5] characterised the reversible maps and obtained the remarkable result that all invertible maps are the composition of at most two reversible maps.

The purpose of the present paper is to give an expository account, putting Jarczyk's results in the context of the conjugacy classification of the real homeomorphism group. This classification may be explained succinctly in terms of a suitable signature concept, which we introduce and explain. We claim no novelty; the classification belongs to folklore (cf. [3, Section(4.2)]), and presumably was known to people such as Milnor and Thurston long ago. However, a concise and accessible account may be useful.

Let $H$ be the group of homeomorphisms of $\mathbb{R}$ onto $\mathbb{R}$, under composition. We denote the identity map by $\mathbb{1}$, and the unary minus map by - . The subgroup $H^{+} \unlhd H$ of orientation-reversing elements has index 2, and the second coset is $H^{-}=H^{+} \circ(-)=(-) \circ H^{+}$.

In Section 2, we describe the conjugacy classes of $H$.

Apart from $\mathbb{1}$, all the elements of finite order in $H$ have order 2 , and belong to $H^{-}$. There are many of these direction-reversing,

proper involutions. In fact, each homeomorphism $\tau_{0}:[0,+\infty)$ onto itself determines a unique involution $\tau \in H^{-}$, defined by

$$
\tau(x)=\left\{\begin{aligned}
-\tau_{0}(x) & , \quad x \geq 0 \\
\tau_{0}^{\circ-1}(-x) & , \quad x<0 .
\end{aligned}\right.
$$

All involutions in $H^{-}$arise in this way.

Let $I$ denote the set of involutions belonging to $H$ (including $\mathbb{1}$ ), and for $n \in \mathbb{N}$, let

$$
I^{\circ n}=\left\{\tau_{1} \circ \cdots \circ \tau_{n}: \tau_{i} \in I\right\}
$$

be the set of maps expressible as the composition of $n$ involutions. By definition, the reversible maps are the elements of $I^{\circ 2}$.

In Section 3, we describe $I^{\circ}$, for each $n$.

In Section 4 we discuss the conjugacy classes in some closelyrelated homeomorphism groups, notably the subgroups that fix a particular subset.

In Section 5 we also describe the compositions of involutions in the related group of invertible germs of continuous functions. 


\section{Conjugacy}

2.1. Signatures. In this section we set notation and describe the conjugacy classes of $H$. The results given belong to folklore at least as far as Lemma (2.3).

To avoid confusion, we write $\phi^{\circ n}$ for the $n$-th compositional power of an element $\phi \in H$.

We write $\operatorname{deg}(\phi)$ for the degree of $\phi\left(+1\right.$ for $\phi \in H^{+}$, and -1 for $\left.\phi \in H^{-}\right)$.

Let $S$ denote the space $\{-1,0,1\}$ of signs, and the function sign : $\mathbb{R} \rightarrow S$ be given by

$$
\operatorname{sign}(x)=\left\{\begin{aligned}
-1 & , \quad x<0 \\
0, & x=0 \\
1 & , \quad x>0
\end{aligned}\right.
$$

We endow $S$ with the largest topology that makes sign continuous. Thus $\{1\}$ and $\{-1\}$ are open, but $\{0\}$ is not. This topology is not Hausdorff, but is convenient for our purposes.

To each $\phi \in H$, we associate the continuous function

$$
s(\phi):\left\{\begin{aligned}
\mathbb{R} & \rightarrow S \\
x & \mapsto \operatorname{sign}(\phi(x)-x) .
\end{aligned}\right.
$$

Let $\Sigma$ denote the family of all continuous maps $h: \mathbb{R} \rightarrow S$.

One may think of an element $h$ of $\Sigma$ as the specification of a closed set $F=h^{-1}(0)$, and a choice of sign $( \pm 1)$ on each connected component of the complement $\mathbb{R} \sim F$.

Note that $S$ becomes a topological semigroup with the usual product, and acts continuously on $\Sigma$ by multiplication:

$$
(s \cdot h)(x)=s \cdot h(x), \quad \forall x \in \mathbb{R}, \forall s \in S, \forall h \in \Sigma .
$$

We regard $\operatorname{deg}(\phi)$ as an element of $S$, when $\phi \in H$, so that $\operatorname{deg}(\phi) \cdot h$ makes sense (as an element of $\Sigma$ ) whenever $h \in \Sigma$.

It is not difficult to prove the following:

Lemma 2.1. $s\left(H^{+}\right)=\Sigma$.

Proof. In fact, if we are given $s_{0} \in \Sigma$, and asked to produce a $\phi \in H^{+}$ with $s(\phi)=s_{0}$, we observe that

- $s_{0}^{-1}(0)$ has to be the fixed-point set of $\phi$;

- on each connected component (an open interval) $J$ of $s_{0}^{-1}(1)$, the map $\phi$ must conjugate to $x \mapsto x+1$ on $\mathbb{R}$, via an increasing homeomorphism $J \rightarrow \mathbb{R}$; 
- on each connected component (an open interval) of $s_{0}^{-1}(-1)$, the map $\phi$ must conjugate to $x \mapsto x-1$ on $\mathbb{R}$ via an increasing homeomorphism $J \rightarrow \mathbb{R}$.

These properties are all we need, and it is easy to choose a $\phi$ that has them.

By checking cases, it is straightforward to verify the following, which expresses the effect of conjugation on $s(\phi)$ :

Lemma 2.2. Let $\phi, \psi \in H$. Then

$$
s\left(\psi^{\circ-1} \circ \phi \circ \psi\right)=\operatorname{deg}(\psi) \cdot(s(\phi) \circ \psi) .
$$

Motivated by this formula, we say that two maps $s_{1}, s_{2} \in \Sigma$ are topologically-equivalent if there exists $\psi \in H$ such that

$$
s_{2}=\operatorname{deg}(\psi) \cdot\left(s_{1} \circ \psi\right) .
$$

This defines an equivalence relation on $\Sigma$. We call the equivalence classes topological signatures, and we denote the class $[s(\phi)]$ by $\operatorname{sig}(\phi)$.

Informally, one may think of a topological signature as a homeomorphism class of pairs $(\mathbb{R}, F)$, with $F$ closed, and a pattern of signs $( \pm 1)$ on the complement of $F$. One is allowed to 'reverse' the set $F$, but then the pattern of signs must also be reversed.

To give a simple example, in the case where $F$ is a singleton, with two complementary components, the patterns $(+1,+1)$ and $(-1,-1)$ are equivalent, but $(-1,+1)$ is not equivalent to $(+1,-1)$. Formally, the three signatures in question here are $\left[\operatorname{sign}^{2}\right]=\left[-\operatorname{sign}^{2}\right],[\operatorname{sign}]$ and $[-\operatorname{sign}]$. Examples of homeomorphisms having these signatures are $x \mapsto x+x^{2}, x \mapsto 2 x$ and $x \mapsto x / 2$, respectively.

As an even simpler example, letting 1 stand for the constant map from $\mathbb{R}$ to 1 , and similarly for -1 , we have that $[1]=[-1]$ is the signature of any fixed-point-free homeomorphism $\phi \in H$. The class $[0]$ is the signature only of the identity map $\mathbb{1}$.

From the definition, $\operatorname{sig}(\phi)$ is a conjugacy invariant of $\phi$. For $\phi \in H^{+}$, it is essentially the only one:

Lemma 2.3. Let $\phi_{1}, \phi_{2} \in H^{+}$. Then $\operatorname{sig}\left(\phi_{1}\right)=\operatorname{sig}\left(\phi_{2}\right)$ if and only if there exists $\psi \in H$ such that $\psi^{\circ-1} \circ \phi_{1} \circ \psi=\phi_{2}$.

Proof. This is straightforward. Only the 'if' part remains to be proved. To prove it, one first reduces to the case in which $\phi_{1}$ and 
$\phi_{2}$ have the same fixed-point set $F$ and pattern of signs on the complementary intervals $J$. One takes $\psi$ to be the identity on $F$, and builds it up on each separate $J$, by fixing any point $c \in J$ and starting with an arbitrary homeomorphism of $\left[c, \phi_{1}(c)\right)$ onto $\left[c, \phi_{2}(c)\right)$. The formula $\phi_{1} \circ \psi=\psi \circ \phi_{2}$ then dictates the extension of this homeomorphism to the rest of $J$.

For instance, each map with $\operatorname{sig}(\phi)=[\operatorname{sign}]$ is conjugate to $x \mapsto$ $2 x$.

In general, $\operatorname{sig}(\phi)$ may have very complex structure. The set of conjugacy classes has the cardinality of the continuum.

We remark that for $\phi \in H$, the signatures $\operatorname{sig}\left(\phi^{\circ n}\right)$ of all the positive compositional powers are obviously conjugation invariants of $\phi$. However, when $\phi \in H^{+}$, these invariants are all the same (and all the powers are conjugate to one another). By contrast, for $\phi \in H^{-}$, the two invariants $\operatorname{sig}(\phi)$ and $\operatorname{sig}(\phi \circ \phi)$ encode different information. Such $\phi$ have just one fixed point, and $\operatorname{sig}(\phi)=[-\operatorname{sign}]$. The fixed point of $\phi$ is a distinguished fixed point of $\phi \circ \phi$, and conjugation maps this distinguished point to the distinguished point of the conjugate. Moreover,

$$
\phi^{\circ-1} \circ(\phi \circ \phi) \circ \phi=\phi \circ \phi,
$$

so Lemma (2.2) gives

$$
s(\phi \circ \phi)=-s(\phi \circ \phi) \circ \phi, \quad \forall \phi \in H^{-} .
$$

This expresses an antisymmetry in the signature of $\phi \circ \phi$. Indeed, if we denote the fixed point of $\phi$ by $p$, and define $\tau$ by

$$
\tau(x)=\left\{\begin{aligned}
\phi(x) & , \quad x \geq p, \\
\phi^{\circ-1}(x) & , \quad x<p,
\end{aligned}\right.
$$

then $\tau$ is a proper involution (which we may think of as a kind of distorted reflection), and

$$
s(\phi \circ \phi)=-s(\phi \circ \phi) \circ \tau .
$$

If we conjugate by a homeomorphism, then this symmetry is essentially preserved ( $-\tau$ becomes $\psi^{\circ-1} \circ \tau \circ \psi$, which is also involutive).

The direction-reversing homeomorphisms $\phi \in H^{-}$are determined up to conjugation by such antisymmetric signatures. More precisely, let $\Sigma^{\text {odd }}$ denote the family of functions $s \in \Sigma$ for which there exists a proper involution $\tau \in I$ with $s=-s \circ \tau$. Then $\Sigma^{\text {odd }}$ is invariant under topological equivalence, and we have: 
Lemma 2.4. The map $\phi \mapsto \operatorname{sig}(\phi \circ \phi)$ induces a bijection between the conjugacy classes of $\phi \in H^{-}$and the equivalence classes of $\Sigma^{\text {odd }}$ under topological equivalence.

Proof. There are two main points here: (1) two maps $\phi_{1}$ and $\phi_{2}$ belonging to $H^{-}$and having $\left[s\left(\phi_{1} \circ \phi_{1}\right)\right]=\left[s\left(\phi_{2} \circ \phi_{2}\right)\right]$ are necessarily conjugate, and (2) each $s_{0} \in \Sigma^{\text {odd }}$ is an $s(\phi \circ \phi)$ for some $\phi \in H^{-}$. Both parts are proved in much the same way, and in the same way as Lemma (2.3), in that the homeomorphisms that need to be constructed are built up separately on the complementary intervals to the fixed-point set of $\phi \circ \phi$, by starting with arbitrary homeomorphisms on specific half-open subintervals. The main difference is that now there is pairing of the complementary intervals, and the maps are built up simultaneously on the pairs. The map can be specified arbitrarily on a half-open interval $[c, \phi \circ \phi(c)$ contained in one interval of the pair, and is then determined on both intervals of the pair.

We record a special case, that is readily proved directly:

Corollary 2.5. Each proper involution is conjugate to - .

In fact, given a proper involution $\tau$, we may assume it fixes 0 , and then it is conjugated to - by the map $\psi$ defined by:

$$
\psi(x)=\left\{\begin{aligned}
x & , \quad x \geq 0, \\
-\tau(x) & , \quad x<0 .
\end{aligned}\right.
$$

\section{InVOLUTIONS}

Let $\Sigma^{\text {even }}$ denote the set of $s \in \Sigma$ having a reflectional symmetry, i.e. $s \in \Sigma^{\text {even }}$ if and only if there exists an involution $\tau \in I \cap H^{-}$ such that

$$
s=s \circ \tau .
$$

(It is equivalent to require merely that there exist a $\tau \in H^{-}$with this property.)

Membership in $\Sigma^{\text {even }}$ is preserved by topological equivalence, so it is a property of signatures. Thus the property $s(\phi) \in \Sigma^{\text {even }}$ is a conjugacy invariant, for $\phi \in H^{+}$.

The following theorem characterises the compositions of two proper involutions: 
Theorem 3.1. (Jarczyk) Let $\phi \in H^{+}$. Then $\phi \in I^{\circ 2}$ if and only if $s(\phi) \in \Sigma^{\text {even }}$.

Proof. Let $\phi \in I^{\circ 2}$. There are two cases:

$1^{\circ} \phi=\mathbb{1}$. In this case, $s(\phi) \equiv 0$, and obviously belongs to $\Sigma^{\text {even }}$.

$2^{\circ} \phi=\tau_{1} \circ \tau_{2}$, where the $\tau_{i}$ are distinct proper involutions. If $\phi(x)>x$, then

$$
\phi\left(\tau_{2}(x)\right)=\tau_{1}(x)>\tau_{1}(\phi(x))
$$

(since $\tau_{1}$ is direction-reversing), and this equals $\tau_{2}(x)$. Similarly, one has the implications

$$
\phi(x)=x \Rightarrow \phi\left(\tau_{2}(x)\right)=\tau_{2}(x)
$$

and

$$
\phi(x)<x \Rightarrow \phi\left(\tau_{2}(x)\right)<\tau_{2}(x) .
$$

Thus

$$
s(\phi) \circ \tau_{2}=s(\phi)
$$

and so $s(\phi) \in \Sigma^{\text {even }}$.

This proves the 'only if' part.

For the other direction, suppose that $s(\phi) \in \Sigma^{\text {even }}$. Choose an involution $\tau_{2}$ such that $s\left(\phi \circ \tau_{2}\right)=s(\phi)$.

We proceed to construct an involution $\tau_{1}$ such that $\tau_{1} \circ \phi=\phi \circ \tau_{2}$. We take $\tau_{1}=\tau_{2}$ on the fixed-point set $F=s(\phi)^{\circ-1}(0)$ of $\phi$. The involution $\tau_{2}$ pairs each component interval $J$ of $\mathbb{R} \sim F$ with another interval $J^{\prime}$ on the other side of the fixed point of $\tau_{2}$. We pick a point $c \in J$ and define $\tau_{1}$ as an arbitrary homeomorphism of the half-open interval $[c, \phi(c)) \subset J$ onto $\left(\tau_{2}(c), \phi\left(\tau_{2}(c)\right)\right] \subset J^{\prime}$. There is then just one way to extend $\tau_{1}$ to the rest of $J \cup J^{\prime}$, while preserving the identity $\tau_{1} \circ \phi=\phi \circ \tau_{2}$. The map defined in this way on $F$ and its complementary intervals is a continuous involution and behaves as required.

Obviously, $\Sigma^{\text {even }}$ is a proper subset of $\Sigma$, so (as if it needed proving) there are elements of $H^{+}$that do not belong to $I^{\circ 2}$. The composition of three proper involutions belongs to $H^{-}$, so the least possible $n$ that could have $I^{\circ n}=H$ is 4 . This is what happens:

Theorem 3.2. (Jarczyk) (1) Each $\phi \in H^{+}$belongs to $I^{\circ 4}$.

(2) Each $\phi \in H^{-}$belongs to $I^{\circ 3}$.

(3) Thus $I^{\circ 4}=H$. 
Proof. (1) Fix $\phi \in H^{+}$.

In the special case when $\operatorname{sig}(\phi)=[1], \phi$ is conjugate to translation by 1 , and factors as the composition of two reflections. Otherwise, $\phi$ has at least one fixed point.

We may assume that 0 is fixed by $\phi$.

We claim that there exist $\phi_{1}, \phi_{2} \in H^{+}$, such that $\operatorname{sig}\left(\phi_{1}\right)=$ $\left[\operatorname{sign}^{2}\right], \operatorname{sig}\left(\phi_{2}\right)=\left[-\operatorname{sign}^{2}\right]$, and $\phi=\phi_{1} \circ \phi_{2}$.

This will suffice, because, $\left[ \pm \operatorname{sign}^{2}\right]$ are symmetric signatures, and hence each $\phi_{i}$ is the composition of two involutions.

To prove the claim, first take

$$
\phi_{2}(x)=\frac{1}{2} \cdot \min \left\{\phi(x), \phi^{\circ-1}(x)\right\},
$$

whenever $x \in \mathbb{R}$. Then one readily verifies that $\phi_{2} \in H^{+}, \phi_{2}(0)=0$, $\phi_{2}(x)<x / 2$ when $x>0$, and $\phi_{2}(x)<2 x$ when $x<0$. Thus the sign of $\phi_{2}(x)-x$ is negative when $x \neq 0$, and $\operatorname{sig}\left(\phi_{2}\right)=\left[-\operatorname{sign}^{2}\right]$.

Next, take $\phi_{1}=\phi \circ \phi_{2}^{\circ-1}$. Routine calculation shows that $\phi_{1}(x)>$ $2 x$ when $x>0$ and $\phi_{1}(x)<x / 2$ when $x<0$. Thus $\operatorname{sig}\left(\phi_{1}\right)=\left[\operatorname{sign}^{2}\right]$.

By construction, $\phi=\phi_{1} \circ \phi_{2}$, and we are done.

(2) This may be proved by means of a similar trick to (1): Given $\phi \in H^{-}$, one chooses an involution $\tau_{1}$ having the same fixed point as $\phi$, and such that $\tau_{1} \circ \phi$ has signature [sign $\left.{ }^{2}\right]$. This is just a matter of squeezing the graph of $\tau_{1}$ above the graph of $\phi$, in such a way that $\phi(x)$ always exceeds (say) twice the maximum of $\phi(x)$ and $\phi^{\circ-1}(x)$.

\section{Special Signatures}

4.1. Oriented Signatures. We confined attention in the foregoing to conjugacy classes with respect to $H$. One could also consider the conjugacy classes of elements of $H^{+}$in $H^{+}$. This leads to the definition of oriented topological equivalence: we say that $s_{1}$ and $s_{2} \in \Sigma$ are oriented-topologically-equivalent if there exists $\psi \in H^{+}$ such that

$$
s_{2}=s_{1} \circ \psi
$$

We call the corresponding equivalence classes the oriented topological signatures. Obviously, each topological signature is the union of one or two oriented topological signatures (depending on whether or not it is symmetric). One may also see that the $H^{+}$-conjugacy classes of elements of $\mathrm{H}^{+}$are in bijective correspondence with the oriented topological signatures. 
It is perhaps worth remarking that the signatures introduced here provide a ready tool for the classification of the normal subgroup structure of $H$. We confine ourselves to a brief summary.

By the support of a function $s \in \Sigma$, we mean the complement in $\mathbb{R}$ of the interior of $s^{\circ-1}(0)$. We denote it by $\operatorname{spt}(\phi)$. We name three subsets of $H$ :

$$
\begin{aligned}
H_{a} & =\{\phi \in H: \operatorname{spt}(\phi) \text { is bounded above }\} \\
H_{b} & =\{\phi \in H: \operatorname{spt}(\phi) \text { is bounded below }\} \\
H_{c} & =\{\phi \in H: \operatorname{spt}(\phi) \text { is bounded }\}
\end{aligned}
$$

These are all subgroups, and contained in $H^{+}$. One has:

Theorem 4.1. (1) $H_{c}$ and $H^{+}$are the only proper normal subgroups of $H$.

(2) $H_{a}, H_{b}$ and $H_{c}$ are the only proper normal subgroups of $H^{+}$.

(3) $H_{c}$ is simple.

The proof involves techniques similar to those already used, and a couple of facts about $s$-functions:

1. Inverses have opposite $s$-functions: $s\left(\phi^{\circ-1}\right)=-s(\phi)$;

2. For homeomorphisms $\phi_{1}, \phi_{2} \in H^{+}$, there is no tidy rule about the $s$-function of a composition, in general, but if $s\left(\phi_{1}\right) \geq 0$ and $s\left(\phi_{2}\right) \geq 0$, then

$$
s\left(\phi_{1} \circ \phi_{2}\right) \geq \max \left\{s\left(\phi_{1}\right), s\left(\phi_{2}\right)\right\} .
$$

These facts are useful because a normal subgroup $N$ of $H$ (respectively, $\left.H^{+}\right)$corresponds to a family $\Sigma(N)$ of $s$-functions closed under the operation

$$
(s(\phi), s(\psi)) \mapsto s\left(\phi^{\circ-1} \circ \psi\right),
$$

and also closed under topological equivalence (resp., oriented topological equivalence).

Fact 1 implies that, given $\phi \in H$ (resp., $H^{+}, H_{c}$ ), there exist $\phi_{1}$ conjugate to $\phi$ and $\phi_{2}$ conjugate to $\phi^{\circ-1}$ (in $H, H^{+}, H_{c}$, resp.) such that $s\left(\phi_{1} \circ \phi_{2}\right)$ is $s(\phi)^{2}$, and hence nonnegative.

Fact 2 allows us to prove that each $\Sigma(N)$ has as an element the characteristic function of some open interval (empty, bounded, semiinfinite, or all of $\mathbb{R}$ ), and this easily yields the result.

We omit the details. 
4.2. $E$-fixed Signatures. If $E \subset \mathbb{R}$ is a fixed closed set, then we may consider the subgroup

$$
H^{\mathrm{fix} E}=\{\phi \in H: \phi(x)=x, \forall x \in E\},
$$

consisting of all homeomorphisms that fix each point of $E$. We denote $H^{\mathrm{fix} E} \cap H^{+}$by $H^{\mathrm{fix} E^{+}}$. Evidently, if $E$ has at least two points, then $H^{\text {fix } E} \leq H^{+}$and $H^{\text {fix } E^{+}}=H^{\text {fix } E}$.

The $s$-functions $s(\phi)$, corresponding to $\phi \in H^{\mathrm{fix} E}$, belong to the set

$$
\Sigma^{E}=\{h \in \Sigma: h(x)=0, \forall x \in E\} .
$$

We say that $h_{1}$ and $h_{2} \in \Sigma^{E}$ are $E$-fixed-topologically-equivalent if there exists $\psi \in H^{\text {fix } E}$ with

$$
h_{1}=\operatorname{deg}(\psi) \cdot h_{2} \circ \psi \text {. }
$$

We call the equivalence classes of this equivalence relation the $E$ fixed topological signatures. Lemma(2.3) is the case $E=\emptyset$ of the following more general result:

Lemma 4.2. Let $E \subset \mathbb{R}$ be closed. Then two elements $\phi_{1}$, $\phi_{2}$ of $H^{f i x E^{+}}$are conjugate in $H^{f i x E}$ if and only if they have the same $E$ fixed topological signature.

The proof involves no new ideas, and is omitted.

When $E$ is nonempty, the group $H^{\text {fix } E^{+}}$embeds naturally as a subgroup of a cartesian product of copies of $\mathrm{H}^{+}$, one for each component of $\mathbb{R} \sim E$. Looking at it this way, one can see that there may be many normal subgoups of $H^{\text {fix } E^{+}}$, in general. In fact, there is a bijective correspondence between the family of normal subgroups and the set of all maps $\nu: \mathbb{R} \rightarrow\left\{\langle\mathbb{1}\rangle, H_{a}, H_{b}, H_{c}, H\right\}$ such that $\nu=\langle\mathbb{1}\rangle$ on $E$, and $\nu$ is constant on each connected component of $\mathbb{R} \sim E$.

\section{Germs of Homeomorphisms}

Let $\mathcal{H}$ denote the group of germs at 0 of elements of $H$ that fix 0. This is the same as the set of germs at 0 of continuous functions $f: \mathbb{R} \rightarrow \mathbb{R}$ that fix 0 and have a compositional inverse on some neighbourhood of 0 . Let $\mathcal{H}^{ \pm}$denote the germs of elements of $H^{ \pm}$ that fix 0 . The involutions in the group $\mathcal{H}$ are exactly the germs from $I$ that fix 0 , and we denote the set of these by $\mathcal{I}$. Let $\Sigma_{0}$ denote the space of germs at 0 of elements of $\Sigma$ that vanish at 0 . Thus an element of $\Sigma_{0}$ is essentially a specification of the germ of a 
closed set $F$, with $0 \in F$, and an 'eventual pattern' of signs on the complement of this germ. Similarly, let $\Sigma_{0}^{\text {odd }} \subset \Sigma_{0}$ and $\Sigma_{0}^{\text {even }} \subset \Sigma_{0}$ denote the germs from $\Sigma^{\text {odd }}$ and $\Sigma^{\text {even }}$, respectively. We define the concept of local topological equivalence of germs of elements of $\Sigma_{0}$ in the obvious way, and we call the classes local signatures.

By moving back and forth between germs and functions representing those germs, and applying the previous results, we deduce the following facts about the involution-structure of the group $\mathcal{H}$.

Theorem 5.1. (1) There is a bijection between the conjugacy classes of $\mathcal{H}^{+}$in $\mathcal{H}$ and the local signatures, induced by $(\phi) \mapsto[\operatorname{germ}(s(\phi))]$.

(2) There is a bijection between the conjugacy classes of $\mathcal{H}^{-}$in $\mathcal{H}$ and the local signatures of elements of $\Sigma_{0}^{\text {odd }}$, induced by

$$
(\phi) \mapsto[\operatorname{germ}(s(\phi \circ \phi))] .
$$

(3) Each proper involution is conjugate to the germ of $x \mapsto-x$.

(4) A germ $g \in \mathcal{H}^{+}$is the composition of two proper involutions if and only if the local signature of $g$ belongs to $\Sigma_{0}^{\text {even }}$.

(5) Each germ belonging to $\mathcal{H}^{-}$is the composition of three proper involutions.

(6) Each germ belonging to $\mathcal{H}^{+}$is the composition of four proper involutions.

It is worth remarking that $\mathcal{H}$ is isomorphic to the quotient group $H / H_{c}$, and this provides another way to retrieve these results.

Finally, we note that combining the ideas of this and the previous section, one may classify the conjugacy classes of the subgroup

$$
\left\{\phi \in H^{+}: \phi(x)=x \text { on some neighbourhood of } E\right\},
$$

whenever $E \subset \mathbb{R}$ is closed, by using "oriented fixed-near- $E$ topological signatures", in an obvious sense.

Acknowledgment. I am grateful to John Murray for useful discussions.

\section{REFERENCES}

[1] Dymphna Graham, Siobhán Keane, and Anthony G.O'Farrell, Siméadracht amchúlaithe chórais dinimiciúil, in: R.N. Shorten, T. Ward and T. Lysaght (eds), Proceedings of the Irish Systems and Signals Conference 2001, pp. $27-31$.

[2] Robert L. Devaney, Reversible diffeomorphisms and flows, Trans. Amer. Math. Soc. 218 (1976) 89-113. 
[3] Greg Hjorth, Classification and Orbit Equivalence Relations, Mathematical Surveys and Monographs, 75. Amer. Math. Soc., Providence, RI, 2000.

[4] Witold Jarzcyk, Reversible interval homeomorphisms, J. Math. Anal. Appl. 272 (2002), 473-479.

[5] Witold Jarzcyk, Reversibility of interval homeomorphisms without fixed points, Aequationes Mathematicae 63 (2002), 66-75.

Anthony G. O'Farrell,

Mathematics Department,

NUI, Maynooth,

Co. Kildare,

Ireland

admin@maths.may.ie

Received on 11 November 2004. 